\title{
Tevatron B-Physics: Recent Results and Prospects
}

\author{
V. Papadimitriou ${ }^{\mathrm{a}}$ \\ (for the CDF and D0 collaborations) \\ aTexas Tech University, Physics Department \\ Lubbock, TX, 79409-1051, U.S.A
}

Between 1992 and 1996, the CDF and D0 experiments have collected data samples of $110 p b^{-1}$ each of $p \bar{p}$ collisions at $\sqrt{s}=1.8 \mathrm{TeV}$ at the Fermilab Tevatron. In the year 2001 the Tevatron commenced $p \bar{p}$ collisions again at $\sqrt{s}=1.96 \mathrm{TeV}$ with the goal of delivering an integrated luminosity of $1 \mathrm{fb}^{-1}$ per year. In the mean time the CDF and D0 detectors have undergone substantial upgrades which allow for a rich $B$ physics program with unique capabilities. In this paper we discuss recent results and the $B$ Physics prospects at the Tevatron with 2 $\mathrm{fb}^{-1}$ of data (Run IIa) or $15 \mathrm{fb}^{-1}$ of data (Run IIa+Run IIb).

\section{INTRODUCTION}

In this paper we discuss the $B$ Physics prospects for the CDF and D0 experiments at the Fermilab Tevatron in Run II. From August 1992 to February 1996, the CDF and D0 detectors collected a data sample of $110 p b^{-1}$ each of $p \bar{p}$ collisions at $\sqrt{s}=1.8 \mathrm{TeV}$ and we refer to this period as Run I. The Tevatron commenced $p \bar{p}$ collisions again at $\sqrt{s}=1.96 \mathrm{TeV}$ in the Spring of 2001 with an initial goal of delivering an integrated luminosity of $1 \mathrm{fb}^{-1}$ per year, corresponding to approximately $10^{11} b \bar{b}$ pairs produced per year. This current data taking period is referred to as Run II. The first phase of Run II is expected to last about three years yeilding a data sample of $2 \mathrm{fb}^{-1}$. Ultimately a data sample of approximately $15 \mathrm{fb}^{-1}$ is expected to be collected before the turn-on of the LHC at $\sim 2007$.

The main goals of the $B$ Physics program at CDF and D0 for Run II are to provide precision measurements of the angles $\beta$ and $\gamma$ of the Unitarity Triangle as well as to exploit the $B_{s}^{0}$ and $B_{c}^{+}$mesons and $b$ baryons which are currently a unique feature of hadron colliders. CDF and D0 will also attempt to provide a measurement of the angle $\alpha$ of the Unitarity Triangle. A precision measurement of $B_{s}^{0}$ flavor oscillations will be very important for testing the unitarity of the Cabibbo-Kobayashi-Maskawa (CKM) mix- ing matrix and the exploration of $\mathrm{CP}$ violation in $B_{s}^{0}$ decays could manifest Physics beyond the Standard Model.

This paper is organized as follows. In section 2 we describe the Run II environment; in section 3 we present the prospects for the measurement of the angles of the Unitarity Triangle and of $B_{s}^{0}$ flavor oscillations. We also present plans for further studies of the $B_{s}^{0}$ meson as well as studies of the $B_{c}^{+}$system and of rare $B$ decays that are sensitive to new physics. Finally in section 4 we present our conclusions.

\section{Run II environment}

The rate of $b \bar{b}$ production at the Tevatron is considerably high, approximately $100 \mu b$, and this, together with the fact that all $b$ species are produced at the Tevatron, makes it a unique place for the study of $b$ production and decay. Although the $b \bar{b}$ production cross section is only one part per thousand of the inelastic cross section, the CDF experiment has shown [1] that exclusive $B$ channels can be succesfully reconstructed in a harsh hadron environment.

The new crucial accelerator components for Run II are the Main Injector, a new $150 \mathrm{GeV}$ accelerator which injects protons and antiprotons into the Tevatron, and the Recycler which is an $8 \mathrm{GeV}$ ring of permanent magnets which will col- 
lect the antiprotons after a store and re-use them. The Main Injector has been commissioned successfully and is expected to increase the rate of production of antiprotons by at least a factor of three above previous rates. The Recycler, to be used later in the Run, is in the commissioning phase. Detector issues are driven by the luminosity, the number of bunches and the time between crossings of the protons and antiprotons. In Run I the crossing time was $3.5 \mu \mathrm{s}$ for 6 bunches and the typical luminosity of order $10^{31} \mathrm{~cm}^{-2} \mathrm{sec}^{-1}$. The crossing times that are expected in Run II are currently $396 \mathrm{~ns}$ for 36 bunches and later $132 \mathrm{~ns}$ for 103 bunches. The corresponding expected typical luminosities are $0.86 \times 10^{32} \mathrm{~cm}^{-2} \mathrm{sec}^{-1}$ and $1-2 \times 10^{32} \mathrm{~cm}^{-2} \mathrm{sec}^{-1}$ with peak luminosities of $2.0 \times 10^{32} \mathrm{~cm}^{-2} \mathrm{sec}^{-1}$ and $4.0 \times 10^{32} \mathrm{~cm}^{-2} \mathrm{sec}^{-1}$ respectively. So far, the typical luminosities are $\sim 10^{31} \mathrm{~cm}^{-2} \mathrm{sec}^{-1}$.

At CDF [2] and D0[3] the detector changes are to the tracking systems, to the calorimeters, to the muon systems, to the front-end electronics and the trigger electronics. In addition, D0 added a new 2 Tesla solenoidal superconducting magnet and CDF improved its particle identification capabilities.

CDF has constructed a new central wire drift chamber (COT), which has a drift time that is reduced by a factor of eight from Run I. $d E / d x$ information for the tracks is also provided by the COT. There is also a new 7 layer (8 layers for $1<|\eta|<2$ ) silicon system in a barrel geometry that extends from a radius of $r=1.6$ $\mathrm{cm}$ from the beam line to $r=28 \mathrm{~cm}$. The layer closest to the beam pipe is a radiation-hard, single-sided detector called Layer 00 which employs recent LHC designs for sensors supporting high bias-voltages. This enables good signal-tonoise performance even after extreme radiation doses. The remaining seven layers are radiationhard, double-sided detectors. This system allows track reconstruction in three dimensions and is expected to have an impact parameter resolution better than $30 \mu \mathrm{m}$ for tracks with transverse momentum of $1 \mathrm{GeV} / \mathrm{c}$. CDF has also replaced the gas calorimeters in the pseudorapidity region $|\eta| \geq 1.0$ with a new scintillating tile calorimeter. In the muon system some gups are closed in the azimuthal coverage for $|\eta| \leq 1.0$ and a new muon system has been built which is covering the region $1.0 \leq|\eta| \leq 1.5$. Finally a Time-of-flight (TOF) detector with expected resolution of $100 \mathrm{ps}$ has been built which will allow a $2 \sigma \mathrm{K} / \pi$ separation for track momentum of less than $1.6 \mathrm{GeV} / \mathrm{c}$.

D0 has constructed a new scintillating fiber tracker and a new silicon vertex detector with 4 barrel layers and disks which extend the tracking up to $|\eta|=2.5$. New scintillating strip electromagnetic preshower detectors have been also built for the central and forward regions to help with the electron identification. D0 has improved as well its muon detection system in general, and has completely rebuilt its forward muon coverage.

To accomodate a 132 ns bunch-crossing time both experiments have essentially replaced all the front end electronics. CDF and D0 have had significant data acquisition and trigger updates as well.

Both experiments use versions of the SVX readout chip. CDF uses un upgraded version of the SVX chip (dead timeless readout) which allows a $50 \mathrm{kHz}$ bandwidth into the Level 2 vertex trigger. This trigger is crucial for efficient triggering on two body decay modes of the $B$ meson such as $B^{0} \rightarrow \pi^{+} \pi^{-}$and the $B_{s}^{0}$ decay modes like $B_{s}^{0} \rightarrow D_{s}^{-} \pi^{+}$and $B_{s}^{0} \rightarrow D_{s}^{-} \pi^{+} \pi^{-} \pi^{+}$. At CDF, fast tracking is now available at Level 1 with the XFT track processor which can find tracks of transvere momentum $p_{T}>1.5 \mathrm{GeV} / \mathrm{c}$ with good momentum and azimuthal resolutions. Information from the silicon detectors is available at Level 2. The SVT trigger processor [2] associates clusters formed from axial strips in the silicon detectors with tracks of $p_{T}>2 \mathrm{GeV} / \mathrm{c}$ found by the $\mathrm{XFT}$, providing a measurement of the impact parameter of the track in the plane transverse to the beam axis. This way it becomes possible to trigger on tracks originating from the decay of longlived $b$ hadrons and therefore coming from vertices different than the primary vertex of the $\bar{p} p$ collision. This allows triggering on "all-hadronic" decays of $b$ hadrons which are important for $B_{s}^{0}$ mixing studies and for the measurement of $\mathrm{CP}$ violation.

In Fig. 1 we show the $D^{+}$and $D_{s}^{+}$mass distributions based on the two-track trigger selection 
using the SVT at CDF.

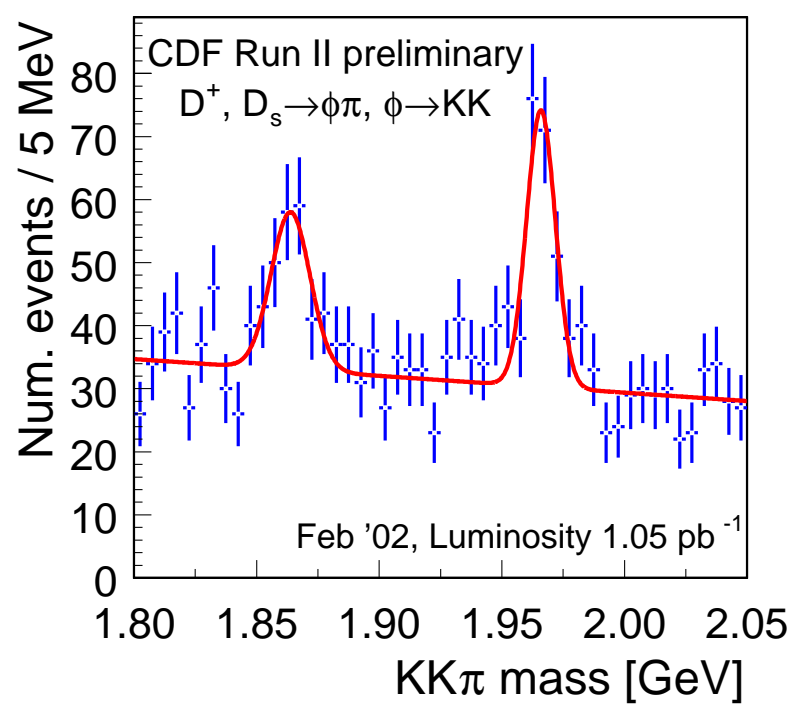

Figure 1. $D^{+}$and $D_{s}^{+}$mass distribution in 1.05 $p b^{-1}$ of Run IIa data at CDF.

\section{3. $B$ Physics Expectations for Run II}

CDF and D0 will address many important questions in $B$ Physics in Run II. Many of the relevant measurements have already been investigated by CDF using the Run I data. In the following subsections we summarize some recent results and the expectations for Run II.

\subsection{Measurement of $\sin (2 \beta)$}

In the $B$ system the measurements of $\mathrm{CP}$ violation that are related ( without large theoretical uncertainties) to angles of the Unitarity Triangle are from asymmetries in the decays of neutral $B$ mesons to $\mathrm{CP}$ eigenstates. For the measurement of the angle $\beta$ the most popular mode is $B^{0} / \overline{B^{0}} \rightarrow J / \psi K_{s}^{0}$. CP violation is expected to manifest itself as an asymmetry in the particle de- cay rate versus antiparticle decay rate to $J / \psi K_{s}^{0}$ :

$$
A_{C P}=\frac{N\left(\bar{B}^{0} \rightarrow J / \psi K_{s}^{0}\right)-N\left(B^{0} \rightarrow J / \psi K_{s}^{0}\right)}{N\left(\bar{B}^{0} \rightarrow J / \psi K_{s}^{0}\right)+N\left(B^{0} \rightarrow J / \psi K_{s}^{0}\right)}
$$

where $N\left(\overline{B^{0}} \rightarrow J / \psi K_{s}^{0}\right)$ is the number of mesons decaying to $J / \psi K_{s}^{0}$ that were produced as $\bar{B}^{0}$ and $N\left(B^{0} \rightarrow J / \psi K_{s}^{0}\right)$ is the number of mesons decaying to $J / \psi K_{s}^{0}$ that were produced as $B^{0}$. In the Standard Model the CP asymmetry in this decay mode is proportional to $\sin 2 \beta$ : $A_{C P}(t)=$ $\sin 2 \beta \sin \left(\Delta m_{d} \mathrm{t}\right)$, where $\beta$ is the angle of the Unitarity Triangle and $\Delta m_{d}$ is the mass difference between the heavy and light $B^{0}$ eigenstates. Even though the time integrated asymmetry can be used to extract $\sin 2 \beta$, measuring the asymmetry as a function of proper decay time is more advantageous. For the measurement of $A_{C P}(t)$ we need to reconstruct the decay mode $B^{0} / \bar{B}^{0} \rightarrow J / \psi K_{s}^{0}$ with good signal-to-background ratio, measure the proper decay time $\mathrm{t}$ and determine whether the meson that decayed was produced as a $B^{0}$ or as $\overline{B^{0}}$. This last component is known as " $b$ flavor tagging". The performance of the $b$ flavor tags can be quantified by their efficiency $\epsilon$ and dilution $D$. The efficiency is the fraction of $B$ candidates to which the flavor tag can be applied. The dilution is related to the probability $P$ that the tag is correct: $D=2 P-1$, that is, a perfect tag has $D=1$ and a random tag has $D=0$. The experimentally measured asymmetry or observed asymmetry is reduced by the dilution of the tag: $A_{C P}^{o b s}=D A_{C P}$. The uncertainty in the asymmetry is inversely proportional to the square root of $\epsilon D^{2} N$ where $N$ is the number of events before the flavor tagging. The product $\epsilon D^{2}$ is usually called "flavor tag effectiveness". The CDF experiment using the entire Run I data of $B^{0} / \bar{B}^{0} \rightarrow$ $J / \psi K_{s}^{0} ; J / \psi \rightarrow \mu^{+} \mu^{-}$decays and three tagging algorithms (same-side tagging, soft-lepton tagging and opposite-side tagging) has found that $\sin 2 \beta=0.79{ }_{-0.44}^{+0.41}$ (stat.+syst.) [4]. CDF has recently updated this measurement. The new measurement is based on $402 \pm 37 B^{0} / \overline{B^{0}} \rightarrow J / \psi K_{s}^{0}$; $J / \psi \rightarrow \mu^{+} \mu^{-}, K_{s}^{0} \rightarrow \pi^{+} \pi^{-}$events and $60 \pm 19$ $B^{0} / \bar{B}^{0} \rightarrow \psi(2 S) K_{s}^{0} ; \psi(2 S) \rightarrow \mu^{+} \mu^{-}, J / \psi \pi^{+} \pi^{-}$ $K_{s}^{0} \rightarrow \pi^{+} \pi^{-}$events. In addition, the oppositeside jet tag has been improved by adding to the 


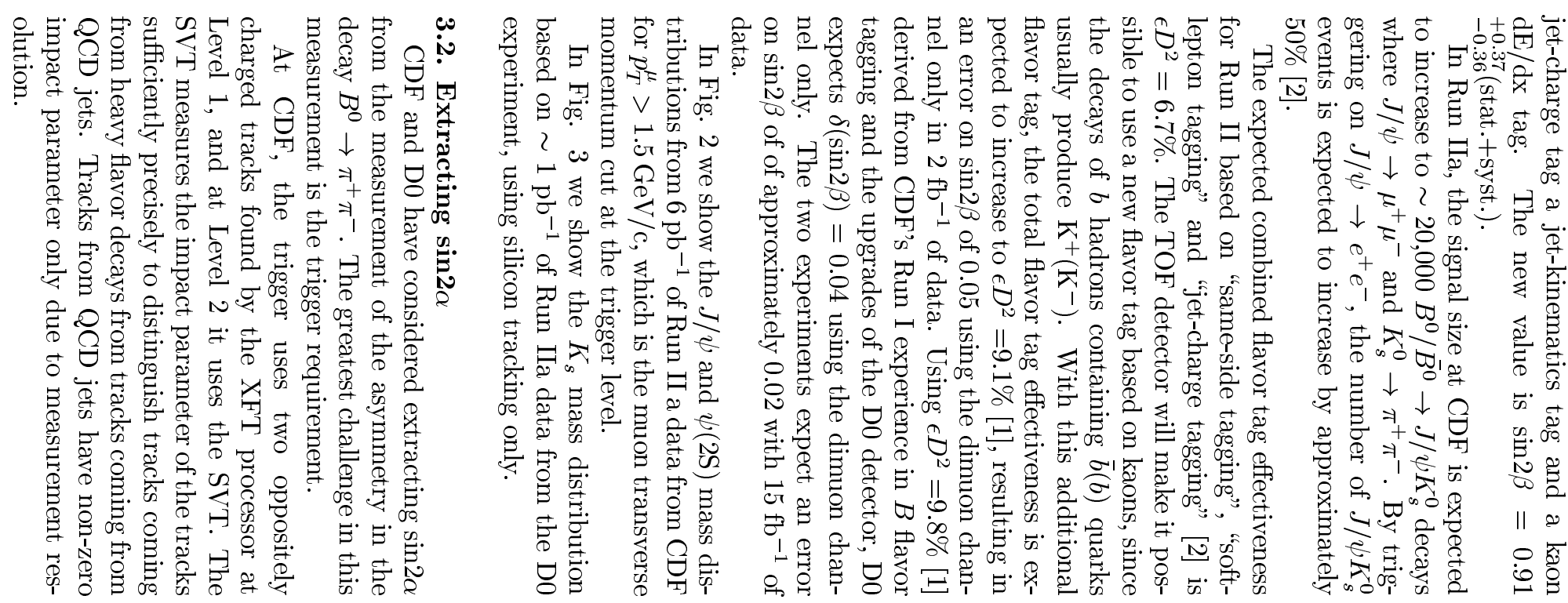

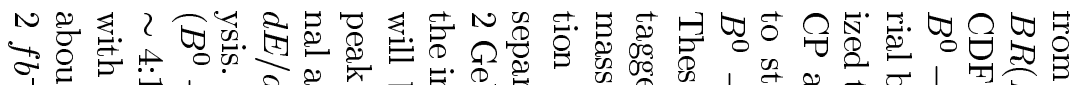
.

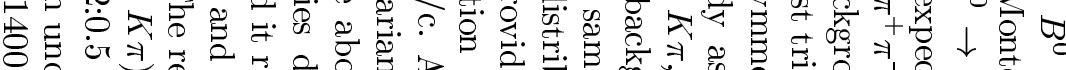

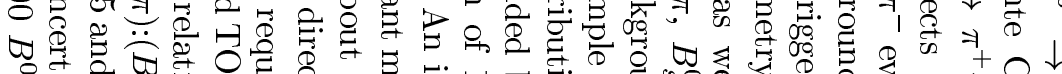

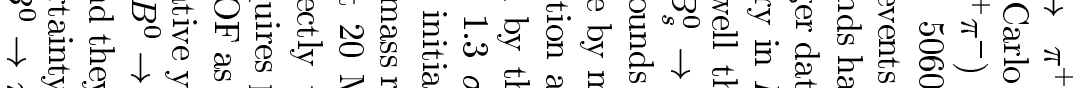

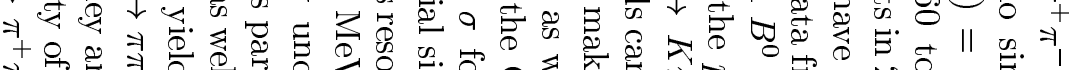

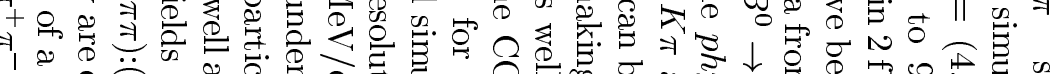

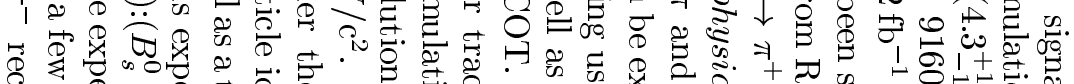

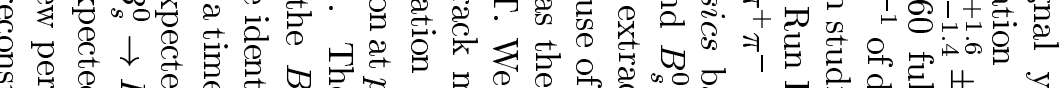

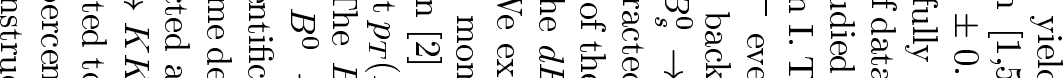

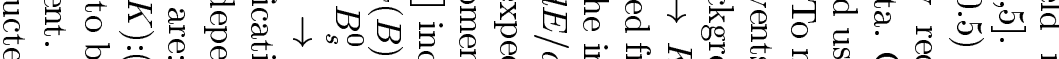

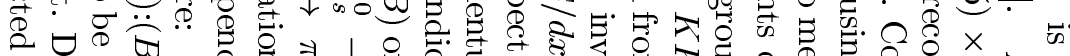

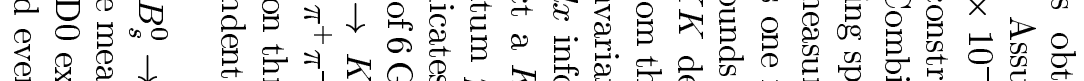

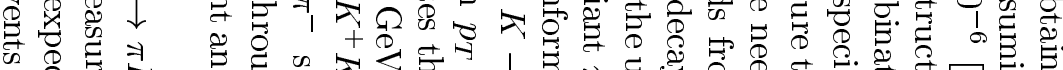

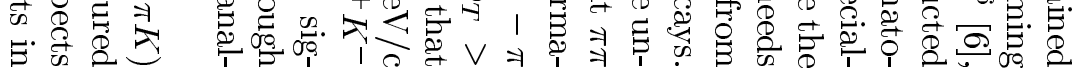

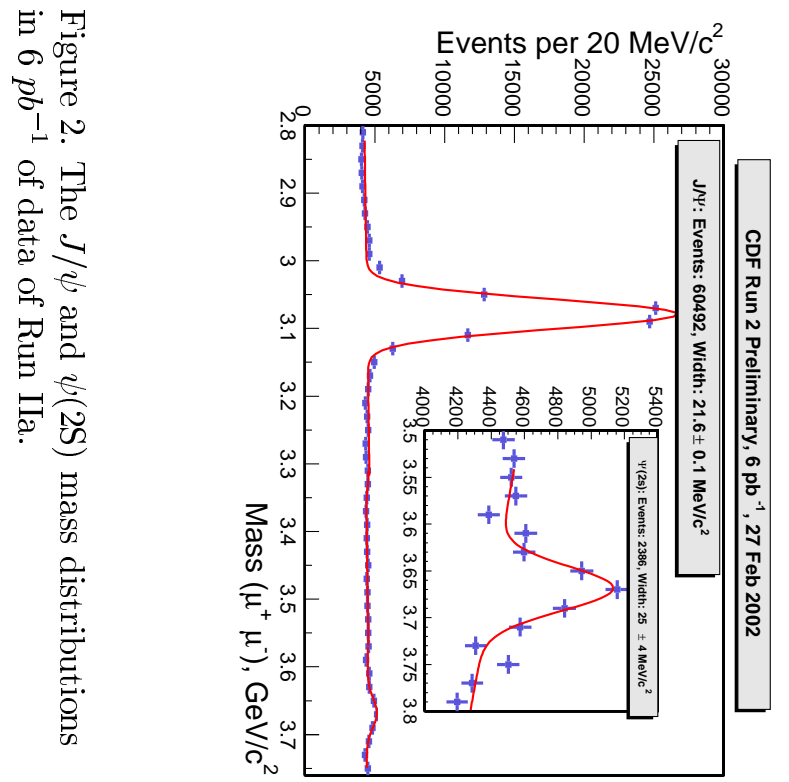




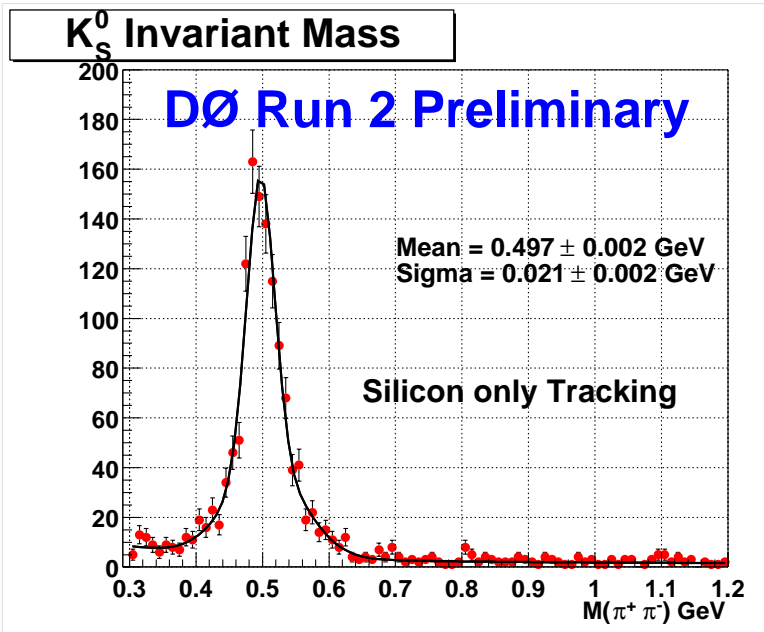

Figure 3. $K_{s}$ mass distribution with the D0 detector in $\sim 1 p b^{-1}$ of Run IIa data.

The final issue related to the extraction of the angle $\alpha$ from the measured time dependence of the $\mathrm{CP}$ asymmetry is the extraction of possible penguin contributions, in addition to the tree level diagrams, requiring theoretical input. As discussed in reference [7], the decay $B^{0} \rightarrow \rho \pi^{0} \rightarrow$ $\pi^{+} \pi^{-} \pi^{0}$ provides enough observables to determine $\alpha$ from an analysis of the time-dependent three-pion Dalitz plot. This method provides a promising alternative way to determine $\sin 2 \alpha$, however, the reconstruction of low momentum $\pi^{0}$ mesons from their decays into two photons will be very challenging for CDF in Run II.

\subsection{Prospects for $B_{s}^{0}-\bar{B}_{s}^{0}$ mixing}

In the Standard Model, $B_{s}^{0} \overline{B_{s}^{0}}$ oscillations occur dominantly through top quark contributions to the electroweak box diagram. The size of the mixing is expressed in terms of the parameter $x_{s}$ which is related to the mass difference between the two mass eigenstates and the average lifetime of the states by $x_{s}=\Delta m_{s} \tau\left(B_{s}^{0}\right)$. The value of $x_{s}$ depends on the top quark mass, the $B_{s}$ decay constant, the QCD bag parameters and corrections due to the breaking of $\mathrm{SU}(3)$ flavor symmetry.
A precision measurement of $B_{s}^{0}$ flavor oscillations, combined with existing measurements of $B^{0}$ oscillations, will be very important for testing the unitarity of the CKM mixing matrix. The combined world average lower limit in $B_{s}^{0}$ flavor oscillations [8] is currently $\Delta m_{s}>14.9$ ps at $95 \%$ C.L. The currently favoured value of the Standard Model for $x_{s}$ [9] is in the range $22.55<x_{s}<34.11$ at $95 \%$ C.L. If the value of $x_{s}$ is small, less than 30 , then both CDF and D0 can use semileptonic decay modes to measure the mixing by early 2003 . If the value of $x_{s}$ is significantly greater, then the exclusive modes will be needed.

The exclusive $B_{s}^{0}$ decay modes used in such studies at $\mathrm{CDF}$ are $B_{s}^{0} \rightarrow D_{s}^{-} \pi^{+}$and $B_{s}^{0} \rightarrow$ $D_{s}^{-} \pi^{+} \pi^{-} \pi^{+}$where the $D_{s}^{-}$is reconstructed as $\phi \pi^{-}, K^{* 0} K^{-}$or $K_{s}^{0} K^{-}$. For the determination of the signal yield it was assumed that $B R\left(D_{s}^{-} \pi^{+}\right)=(0.30 \pm 0.04) \%$ and $B R\left(D_{s}^{-} \pi^{+} \pi^{-} \pi^{+}\right)=(0.80 \pm 0.25) \%$ [5]. The data collection of $B_{s}^{0}$ decay modes is based on the two-track trigger used for the collection of $B^{0} \rightarrow$ $\pi^{+} \pi^{-}$events. CDF expects 75,000 reconstructed $B_{s}^{0}$ decays in $2 \mathrm{fb}^{-1}$ of data using all the above decay modes. For the extraction of the signals from combinatorial background CDF estimates a signal-to-background ratio in the range $1: 2$ to $2: 1$ [5].

Layer 00 and TOF play an important role for the evaluation of the sensitivity to $B_{s}^{0}-\bar{B}_{s}^{0}$ oscillations at $\mathrm{CDF}$. The addition of Layer 00 provides more precise decay length measurements which improves the proper time resolution from $\sigma_{t}=$ $60 \mathrm{fs}$ to $\sigma_{t}=45 \mathrm{fs}$. The TOF system is expected to improve the $\epsilon D^{2}$ flavor tag effectiveness from $5.7 \%$ to $11.3 \%$. Figure 4 shows the integrated luminosity required to achieve a five standard deviation observation of $B_{s}^{0}-\bar{B}_{s}^{0}$ mixing for three different signal-to-background ratios. The curves shown in the figure assume that excellent trigger and reconstruction efficiency was achieved and 75,000 reconstructed $B_{s}^{0}$ decays. They also assume the excellent proper time resolution and flavor tagging efficiency $\mathrm{CDF}$ is aiming for. The $x_{s}$ reach is expected to be 74 for $\mathrm{S} / \mathrm{B}=2: 1$ and 69 for $\mathrm{S} / \mathrm{B}=1: 2$. Therefore, the expected $\mathrm{CDF}$ reach in Run II covers very well the currently favoured values of $x_{s}$ within the Standard Model. 


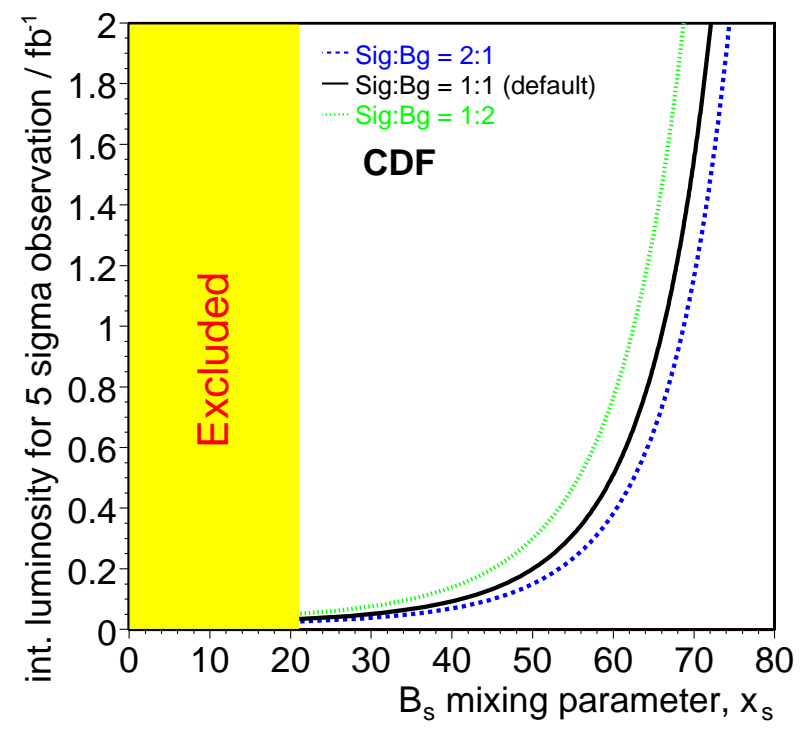

Figure 4. Integrated luminosity required to achieve a five standard deviation observation of $B_{s}^{0}-\overline{B_{s}^{0}}$ mixing.

\subsection{CP asymmetry in $B_{s}^{0} \rightarrow J / \psi \phi$}

While the CP asymmetry in $B^{0} / \bar{B}^{0} \rightarrow J / \psi K_{s}^{0}$ measures the weak phase of the CKM matrix element $V_{t d}$, the CP asymmetry in $B_{s}^{0} / \bar{B}_{s}^{0} \rightarrow J / \psi \phi$ measures the weak phase of $V_{t s}$ which is expected to be very small within the Standard Model. Observing an asymmetry in $B_{s}^{0} / \bar{B}_{s}^{0} \rightarrow J / \psi \phi$ would signal the existence of an anomalous $\mathrm{CP}$ violating phase [7].

On the basis of the Run I experience, CDF expects that the yield of $B_{s}^{0} \rightarrow J / \psi \phi$ in Run II will be about $60 \%$ of the $B_{s}^{0} \rightarrow J / \psi K_{s}$ yield. The flavor tagging efficiency for $B_{s}^{0} \rightarrow J / \psi \phi$ is expected to be $9.7 \%$ with the TOF detector. An angular analysis may be necessary to separate the different possible $\mathrm{CP}$ eigenstates contributing to this final state. If we neglect any loss of sensitivity due to this procedure, with the assumptions stated above and for $x_{s}=25, \mathrm{CDF}$ expects to measure the $\mathrm{CP}$ asymmetry in $B_{s}^{0} \rightarrow J / \psi \phi$ with a resolution of about 0.1 with $2 \mathrm{fb}^{-1}$. A resolution between 0.03 and 0.06 is expected with $15 \mathrm{fb}^{-1}$, depending on the $C P$ content of the final state. This is close to the Standard Model expectation of roughly 0.02 , allowing for a sensitivity to new $C P$-violating physics in this mode.

\subsection{Measurement of the angle $\gamma$}

One of the best tools to extract the angle $\gamma$ are measurements of the time-dependent asymmetries in $B_{s}^{0}$ decays. CDF has considered measuring $\gamma$ using the decays $B_{s}^{0} \rightarrow D_{s}^{-} K^{+}$and $B^{ \pm} \rightarrow D K^{ \pm}$.

Data for the first decay mode will be collected at CDF with the same two-track trigger as for $B^{0} \rightarrow \pi^{+} \pi^{-}$and $B_{s}^{0} \rightarrow D_{s}^{-} \pi^{+}$. In $2 \mathrm{fb}^{-1}$ of data $\mathrm{CDF}$ expects to have about $850 B_{s}^{0} \rightarrow D_{s}^{-} K^{+}$ signal events before flavor tagging. In order to estimate the signal-to-background ratio CDF considered physics backgrounds and combinatorial background. The main physics background is from $B_{s}^{0} \rightarrow D_{s}^{*-} \pi^{+}$decays. Studies of Run I data indicate that the signal to background ratio should be between 0.5 and 2 , not including improvements that may be coming from $d E / d x$ and TOF information and three-dimensional vertexing.

To estimate the CDF reach for $\sin \gamma$, Monte Carlo studies generated pseudo-experiments which included signal, backgrounds, resolution smearing as well as mistagging. Each pseudoexperiment was subjected to a fitter extracting $\sin (\gamma+\delta)$ and $\sin (\gamma-\delta)$ as fit parameters where $\delta$ is the strong phase. Within the first $2 \mathrm{fb}^{-1}$, the expected error on $\sin (\gamma \pm \delta)$ is around 0.4 to 0.7 depending upon what the backgrounds turn out to be. With $15 \mathrm{fb}^{-1}$, an uncertainty near 0.1 may be achievable.

Another way to extract the CKM angle $\gamma$ had been originally suggested by Gronau, London and Wyler [10]. It is based on measuring $B^{ \pm}$decay rates involving $D^{0} / \bar{D}^{0}$ mesons and requires the interference between two amplitudes that are significantly different in magnitude causing the resulting asymmetries to be small. A refinement of this method has been suggested by Atwood, Dunietz and Soni [11] using decays to final states that are common to both $D^{0}$ and $\bar{D}^{0}$ and that are not $C P$ eigenstates. In particular, 
large $C P$ asymmetries can result from the interference of the decays $B^{-} \rightarrow K^{-} D^{0}$ and $B^{-} \rightarrow$ $K^{-} \bar{D}^{0}$ with $D^{0} \rightarrow f$ being a doubly Cabibbo suppressed decay while $\bar{D}^{0} \rightarrow f$ is Cabibbo allowed. The measurement of interference effects in these modes allows the extraction of $\gamma$ without the knowledge of $B R\left(B^{-} \rightarrow K^{-} \bar{D}^{0}\right)$.

In a preliminary study, CDF has investigated the two $D^{0}$ final states $K^{-} \pi^{+}$and $K^{-} \pi^{+} \pi^{-} \pi^{+}$. The $B^{-} \rightarrow K^{-} D^{0}$ data samples would be collected using the two-track hadronic trigger used for $B^{0} \rightarrow \pi^{+} \pi^{-}$or hadronic $B_{s}^{0}$ decays. In $2 \mathrm{fb}^{-1}$, 100 to 140 events are expected to be recorded for $B^{-} \rightarrow K^{-} D^{0}$ with $D^{0} \rightarrow K^{-} \pi^{+}$and about the same number for $D^{0} \rightarrow K^{-} \pi^{+} \pi^{-} \pi^{+}$. The studies indicate that if the combinatoric backgrounds can be controlled and if the necessary branching ratios are known with an uncertainty of $20 \%$, a resolution on $\gamma$ of $\sim 15^{\circ}$ could be possible with $15 \mathrm{fb}^{-1}$.

The angle $\gamma$ can be also measured [1] by relating the $\mathrm{CP}$ violation observables in $B^{0} \rightarrow \pi^{+} \pi^{-}$ and $B_{s}^{0} \rightarrow K^{+} K^{-}$and using U-spin symmetry to relate the ratio of hadronic matrix elements for penguin and tree diagrams. Detailed studies show that by using this technique, $\gamma$ can be measured to $\sim \pm 10^{\circ}$ with a four-fold ambiguity in Run IIa, assuming that $\sin (2 \beta)$ is pecisely known from $B^{0} \rightarrow J / \psi K_{s}^{0}$. By allowing $20 \% \mathrm{SU}(3)$ symmetry breaking, the theoretical uncertainty is expected to be $\sim \pm 3^{\circ}$. With $15 \mathrm{fb}^{-1}$ of data, the statistical uncertainty should be $\sim \pm 3^{\circ}$, making this a very promising method for measuring $\gamma$.

\section{6. $B_{c}^{+}$Mesons and Rare $B$ decays}

We expect three major contributions to the $B_{c}^{+}$decay width: $\bar{b} \rightarrow \bar{c} W^{+}$with the $c$ as a spectator, leading to final states like $J / \psi \pi^{+}$or $J / \psi \ell^{+} \nu ; c \rightarrow s W^{+}$, with the $\bar{b}$ as spectator, leading to final states like $B_{s}^{0} \pi^{+}$or $B_{s}^{0} \ell^{+} \nu$; and $c \bar{b} \rightarrow W^{+}$annihilation, leading to final states like $D K, \tau \nu_{\tau}$ or multiple pions. CDF searched for the decay channels $B_{c}^{+} \rightarrow J / \psi \mu^{+} \nu$ and $B_{c}^{+} \rightarrow$ $J / \psi e^{+} \nu$ followed by $J / \psi \rightarrow \mu^{+} \mu^{-} . \quad 20.4_{-5.5}^{+6.2}$ events were observed in these decay channels and the $B_{c}^{+}$mass and lifetime were measured to be $6.40 \pm 0.39$ (stat.) \pm 0.13 (syst.) $\mathrm{GeV} / \mathrm{c}^{2}$ and $0.46_{-0.16}^{+0.18}($ stat.) \pm 0.03 (syst.) ps respectively[12].
The increase of statistics expected in Run II of the Tevatron Collider (increase in yield by a factor of $\sim 50$ in the $J / \psi$ decay modes for the first 2 $\mathrm{fb}^{-1}$ ), combined with refinement in technique and investigation of additional decay channels for the $B_{c}^{+}$meson, should allow CDF to measure the $B_{c}^{+}$ mass, lifetime and production cross section with much better accuracy than the one achieved with the Run I data. It should also allow to measure ratios of branching ratios of the $B_{c}^{+}$for various decay channels. Let us note here that for CDF the mass uncertainty from the $B_{c}^{+}$hadronic channels involving a $J / \psi$ is about $8 \mathrm{MeV} / \mathrm{c}^{2}$, to be compared with the $411 \mathrm{MeV} / \mathrm{c}^{2}$ uncertainty achieved in Run I with the semileptonic decay modes.

Rare $B$ decays provide a stringent test of the Standard Model for possible new physics effects, such as an anomalous magnetic moment of the $W$ and the presence of a charged Higgs. Experimentally, these rare decays are accessible at CDF via the dimuon trigger, which is one of the most important $B$ physics triggers. CDF has performed a search for the decay modes $B^{ \pm} \rightarrow \mu^{+} \mu^{-} K^{ \pm}$, $B^{0} \rightarrow \mu^{+} \mu^{-} K^{* 0}$ and $B_{d, s}^{0} \rightarrow \mu^{+} \mu^{-}$[13] using Run I data. The sensitivity of Run II CDF for the rare-decay modes $B_{d(s)} \rightarrow K^{* 0} \gamma, \Lambda_{b} \rightarrow \Lambda \gamma$, $B_{d} \rightarrow \mu^{+} \mu^{-} K^{* 0}$ and $B_{d(s)} \rightarrow \mu^{+} \mu^{-}$are:

$$
\begin{gathered}
N\left(B_{d} \rightarrow K^{* 0} \gamma\right)= \\
(170 \pm 50) \times \frac{\int L}{2 f b^{-1}} \times \frac{B r\left(B_{d} \rightarrow K^{* 0} \gamma\right)}{4.5 \times 10^{-5}}
\end{gathered}
$$

$N\left(B_{s} \rightarrow K^{* 0} \gamma\right)=(12 \pm 4) \times \frac{\int L}{2 f b^{-1}} \times \frac{B r\left(B_{d} \rightarrow K^{* 0} \gamma\right)}{4.5 \times 10^{-5}}$

$N\left(\Lambda_{b} \rightarrow \Lambda \gamma\right)=(4.0 \pm 1.7) \times \frac{\int L}{2 f b^{-1}} \times \frac{B r\left(\Lambda_{b} \rightarrow \Lambda \gamma\right)}{4.5 \times 10^{-5}}$

$$
\begin{gathered}
N\left(B_{d} \rightarrow K^{* 0} \mu \mu\right)= \\
(59 \pm 12) \times \frac{\int L}{2 f b^{-1}} \times \frac{B r\left(B_{d} \rightarrow K^{* 0} \mu \mu\right)}{1.5 \times 10^{-6}}
\end{gathered}
$$

$\operatorname{Sensitivity}\left(B_{d} \rightarrow \mu \mu\right)=3.5 \times 10^{-9} \times \frac{2 f b^{-1}}{\int L}$ 


$$
\operatorname{Sensitivity}\left(B_{s} \rightarrow \mu \mu\right)=1.0 \times 10^{-8} \times \frac{2 f b^{-1}}{\int L}
$$

CDF expects to observe $\sim 440 B_{d} \rightarrow \mu^{+} \mu^{-} K^{* 0}$ events with $15 \mathrm{fb}^{-1}$ with the dimuon plus displaced track trigger. This data will most probably allow to study both the invariant mass distribution of the dimuon pair as well as the forwardbackward charge asymmetry in the decay. Both of these distributions are sensitive to physics beyond the Standard Model [14].

\section{Conclusions}

CDF has established a rich and competative $B$ Physics program in Run I and has shown that the study of $B$ Physics is possible in a hadron collider environment. Both CDF and D0 have upgraded detectors with unique capabilities for $B$-Physics measurements in Run II.

The expectations are: measurement of $\sin 2 \beta$ with an uncertainty of 0.04 in Run IIa and 0.02 in Run IIa+IIb; measurement of the $\mathrm{CP}$ aymmetry in the decays $B^{0} \rightarrow \pi^{+} \pi^{-}$and $B_{s} \rightarrow K^{+} K^{-}$; measurement of the angle $\gamma$ to better than $10^{\circ}$ in Run IIa and to about $3^{\circ}$ in Run IIa+IIb; a five standard deviation sensitivity for a $B_{s}^{0}-\bar{B}_{s}^{0}$ mixing measurement up to about $x_{s}=60$ in Run IIa; measurement of the $\mathrm{CP}$ asymmetry in the decay $B_{s}^{0} \rightarrow J / \psi \phi$ with about $10 \%$ uncertainty for $x_{s}=25$ in Run IIa and between $3 \%$ and $6 \%$ in Run IIa+IIb; observation of exclusive $B_{c}^{+}$meson decays; observation of $b$ baryons and of rare $B$ decays.

With these and other measurements that CDF and D0 will pursue in Run II, we expect to impose severe constraints on the Standard Model of weak quark mixing and $\mathrm{CP}$ violation and be sensitive to new physics.

\section{REFERENCES}

1. Pub. Proceedings of the Workshop "B Physics at the Tevatron: Run II and Beyond", FERMILAB-PUB-01/197.

2. R. Blair et al. (the CDF II Collaboration), "The CDF II Detector Technical Design Re- port", Fermilab Pub-96/390-E; see also http://www-cdf.fnal.gov/upgrades/.

3. The D0 Collaboration, "The D0 Upgrade", Fermilab Pub-96/357-E; see also http://www-d0.fnal.gov/hardware/upgrade/.

4. T. Affolder et al., Phys. Rev. D 61, 072005 (2000).

5. "Proposal for Enhancement of the CDF II Detector: An Inner Silicon Layer and a Time of Flight Detector" (P-909); "Update to Proposal P-909: Physics Performance of the CDF II with an Inner Silicon Layer and a Time of Flight Detector". Both these documents can be found at www-cdf.fnal.gov/upgrades/.

6. D. Cronin-Hennessy et al. (CLEO Collaboration), Phys. Rev. Lett. 85, 515 (2000).

7. Y. Nir, Proceedings of "18th International Symposium on Lepton-Photon Interactions (LP 97)", Hamburg, Germany (1997). Also see hep-ph/9709301.

8. The LEP working group on $B$ oscillations: http://www.cern.ch/LEPBOSC/ combined_results/lathuile_2002/.

9. M. Ciuchini, "7th Topical Seminar on the Legacy of LEP and SLC", 8-11 October 2001, Siena (Italy) hep-ph/0112133 (2001).

10. M. Gronau and D. London, Phys.Lett. B253, 483 (1991);

M. Gronau and D. Wyler, Phys.Lett. B265, 172 (1991).

11. D. Atwood, I. Dunietz and A. Soni, Phys.Rev.Lett. 78, 3257 (1997).

12. F. Abe et al., Phys. Rev. Lett. 81, 2432 (1998); F. Abe et al., Phys. Rev. D 58, 112004 (1998).

13. F. Abe et al., Phys. Rev. Lett. 76, 4675 (1996); F. Abe et al., Phys. Rev. D 57, R3811 (1998); T. Affolder et al., Phys. Rev. Lett. 83, 3378 (1999).

14. A. Ali, T. Mannel and T. Morozumi, Phys. Lett. B 273, 473 (1991); B. Grinstein, M.J. Savage and M.B. Wise, Nucl. Phys. B 319, 271 (1989); W. Jaus and D. Wyler, Phys. Rev. D 41, 3405 (1991). 